\title{
Magnetic second-harmonic generation from the terraces and steps of aligned magnetic nanostructures grown on low symmetry interfaces
}

\author{
L Carroll, K Fleischer, J P Cunniffe and J F McGilp ${ }^{1}$ \\ School of Physics, Trinity College Dublin, Dublin 2, Republic of Ireland \\ E-mail: jmcgilp@tcd.ie
}

Received 14 March 2008

Published 22 May 2008

Online at stacks.iop.org/JPhysCM/20/265002

\begin{abstract}
Aligned magnetic nanostructures grown on low symmetry interfaces are generally inhomogeneous, with different magnetic species, such as terrace and step atoms, contributing to the overall magnetic response from the interfacial regions. It is shown that the presence of different magnetic regions can be detected by means of normal incidence (NI) magnetic second-harmonic generation (MSHG). A phenomenological model of NI MSHG at magnetic interfaces of $1 \mathrm{~m}$ symmetry is developed and a methodology is described for optimizing the signal-to-noise ratio of extracted hysteresis curves by adjusting the input polarization angle. Quadratic terms in the magnetization are properly accounted for, using recently published formulae. It is shown that, where more than one magnetic region is present, the shape of the extracted hysteresis curve, which contains contributions from the different magnetic regions, varies with the input polarization angle. The new approach is used to determine hysteresis loops from the various magnetic regions of Au-capped ultrathin Fe films grown on a vicinal W(110) substrate. The results for $0.75 \mathrm{ML}$ Fe coverage are of particular interest, revealing distinct contributions from terrace and step Fe atoms. This experimental procedure and phenomenology opens up low symmetry magnetic interfaces and aligned nanostructures to characterization by means of MSHG.
\end{abstract}

(Some figures in this article are in colour only in the electronic version)

\section{Introduction}

Laterally confined magnetic nanostructures are attracting considerable interest, with ferromagnetism being reported even in single atomic wires of Co grown on $\operatorname{Pt}(997)[1,2]$. In order to be useful, such nanostructures have to be protected from environmental corrosion and contamination, and this is accomplished by capping the structure with a thin protective layer. These buried nanostructures, which are located in the interfacial region between the substrate and capping layer, are not accessible to conventional surface probes. Photonin/photon-out techniques ('epioptics' [3]), such as optical second-harmonic generation (SHG), which use symmetry to

\footnotetext{
1 Author to whom any correspondence should be addressed.
}

extract the optical response of the interface from the normally dominant bulk response, allow the buried interfacial structure of centrosymmetric materials to be probed through thin capping layers, and magnetic SHG (MSHG) extends this to magnetic interfaces. However, the vast majority of magnetic thin film systems that have been studied so far are of high surface and interface symmetry [4], because of the general complexity of the MSHG response and the often poor signalto-noise ratio (SNR) [5].

Lower symmetry systems, which may have multiple magnetic regions, have many tensor components that may contribute to the MSHG intensity, making interpretation particularly difficult. In this paper a new approach to MSHG studies of lower symmetry systems is developed and applied to 
a well-known system, Fe nanostripes on W(110) [6-8], capped with $\mathrm{Au}[9]$.

The smallest magnetic nanostructures have been grown on the aligned atomic steps of vicinal substrates [1,8], which typically show only $1 \mathrm{~m}$ symmetry in the SHG response from the surface or interface, with the single mirror plane orthogonal to the step edge direction [10]. This $1 \mathrm{~m}$ structure is particularly challenging, having 10 allowed crystallographic tensor components that can contribute to the SHG signal, and up to 8 additional magnetic tensor components, for an in-plane magnetic easy axis aligned with the $1 \mathrm{~m}$ mirror plane. However, normal or near-normal incidence (NI) SHG geometry simplifies the nonlinear response from such low symmetry systems by excluding $z$-dependent tensor components.

Within the electric dipole approximation, the intensity of MSHG from a magnetic interface is given by

$$
I(2 \omega ; \pm M) \propto\left|\chi_{\mathrm{even}}^{\mathrm{eff}} E(\omega) E(\omega) \pm \chi_{\mathrm{odd}}^{\mathrm{eff}} M E(\omega) E(\omega)\right|^{2}
$$

where $\chi_{\text {even }}^{\text {eff }}$ is the effective third rank crystallographic susceptibility tensor, $E(\omega)$ is the input electric field vector, $\chi_{\text {odd }}^{\text {eff }}$ is the effective fourth rank axial magnetic susceptibility tensor, and $M$ is the interface magnetization [11]. Any higher order crystallographic quadrupolar contribution from the substrate or capping layer can, in principle, be included in the even term, but the combination of NI and choice of azimuth removes this contribution for the experimental geometries used in this work. SHG is known to be sensitive to strain [12] and any magneto-elastic effects will also appear in the even term [13, 14]. Appropriate Fresnel and local field factors [15] are included in the effective tensors, as NI geometry reduces these contributions to simple scaling factors. It has been shown that, through careful choice of sample alignment with respect to input polarization selection and the direction of applied magnetic field, it is possible to measure a NI MSHG intensity from $1 \mathrm{~m}$ magnetic interfaces containing just one contributory crystallographic tensor component (even with respect to reversal of the magnetization direction) and two magnetic (odd) tensor components [16]. It was recently demonstrated that, with this geometry, the relative magnitude and phase of the even and odd components can be determined [17].

In general, low symmetry magnetic interfaces are inhomogeneous, containing two or more regions where the same magnetic species is found with a different number of magnetic and non-magnetic nearest neighbours. Since the magnetic properties of an interface are known to depend sensitively on nearest neighbour number and type [7], a full description of NI MSHG from an inhomogeneous interface must account for the contributions made by the different regions. In this paper it is shown that fine tuning of the input polarization direction can enhance the magnetic contribution to the NI SHG signal, resulting in a significant improvement in the signal-to-noise ratio (SNR) of hysteresis loops extracted from the measurements. It is also shown that the improved SNR allows the presence of different magnetic regions at the inhomogeneous interface to be identified by changing the input polarization direction, which alters relative contribution of the tensor components to the overall NI MSHG response.

\section{Experiment}

Preparation of the Au-capped ultrathin Fe films has been described in detail elsewhere [18]. Briefly, pseudomorphic Fe films were grown under ultrahigh vacuum conditions (base pressure $<4 \times 10^{-11}$ mbar) on a clean vicinal W(110) single crystal substrate, offcut $1.4^{\circ}$ in the [1ํㅣ $(x)$ direction, which produces atomic steps running in the [001] $(y)$ direction. This offcut was chosen to allow direct comparison with previous work by Elmers and co-workers [8,9]. The films were formed by deposition at room temperature followed by annealing at $800 \mathrm{~K}$ for $3 \mathrm{~min}$ [8]. The Fe films were protected from ex situ contamination by a $16 \mathrm{~nm}$ thick, optically isotropic capping layer of $\mathrm{Au}$, deposited at room temperature. The capped samples were placed in an optical cryostat and the MSHG intensity, $I_{y}\left(2 \omega ; \varphi, \pm M_{X}\right)$, was measured at $80 \mathrm{~K}$ with the applied magnetic field aligned along the [1ㅣㅣㄹ easy axis. The $2 \omega$ electric field vector was aligned along the steps and the input polarization angle, $\varphi$, could be varied. A femtosecond laser system was used as the fundamental frequency light source, tuned to $1.51 \mathrm{eV}$ for 2 and $3 \mathrm{ML} \mathrm{Fe}$ coverage, and $1.55 \mathrm{eV}$ for the 0.25 and $0.75 \mathrm{ML}$ Fe coverage. Calculations predict a strong MSHG response from the Fe films in this spectral region [19]. Unamplified, 130 fs Ti:sapphire laser pulses of average power of $0.9 \mathrm{~W}$ were used, at a repetition rate of $76 \mathrm{MHz}$. The beam size was $40 \mu \mathrm{m}$ at the sample and the angle of incidence was $<3^{\circ}$.

\section{NI MSHG from magnetic interfaces of $1 \mathrm{~m}$ symmetry}

The third rank even crystallographic tensor components and fourth rank odd magnetic tensor components in equation (1) are expressed using the simplified notation $\chi_{i j k} \equiv i j k$ and $\chi_{i j k L} \equiv i j k L$, respectively, where the upper-case subscript $L$, describing the magnetization direction, is introduced to avoid potential confusion with unrelated quadrupolar susceptibility tensor components [4]. The interface formed by the pseudomorphic deposition of a magnetic species on a vicinal surface, consisting of well-ordered $1 \mathrm{~m}$ atomic steps separated by higher symmetry surface terraces, possesses overall $1 \mathrm{~m}$ symmetry. If the surface normal of the interface is in the $z$ direction, the normal to the single mirror plane is in the $y$ direction, and the magnetization is in the $x$-direction, then the $y$-polarized NI MSHG intensity probes only three tensor components, $y x y, y x x X$ and $y y y X$ [16]. The dependence of the $y$-polarized NI MSHG intensity on $\varphi$, the angle between the input polarization field and the $x$-direction, is given by:

$$
\begin{aligned}
& I_{y}\left(2 \omega ; \varphi, \pm M_{X}\right) \propto \mid y x y \sin 2 \varphi \\
& \quad \pm\left.\left\{y x x X \cos ^{2} \varphi+y y y X \sin ^{2} \varphi\right\} M_{X}\right|^{2} .
\end{aligned}
$$

As well as producing these relatively simple expressions, NI geometry has the advantage of eliminating any isotropic capping layer contribution to the even term, and thus also to the phase difference, $\delta$. If the surface of the capping layer is macroscopically isotropic, as is often the case where no special effort is made to grow an epitaxial capping layer, then 

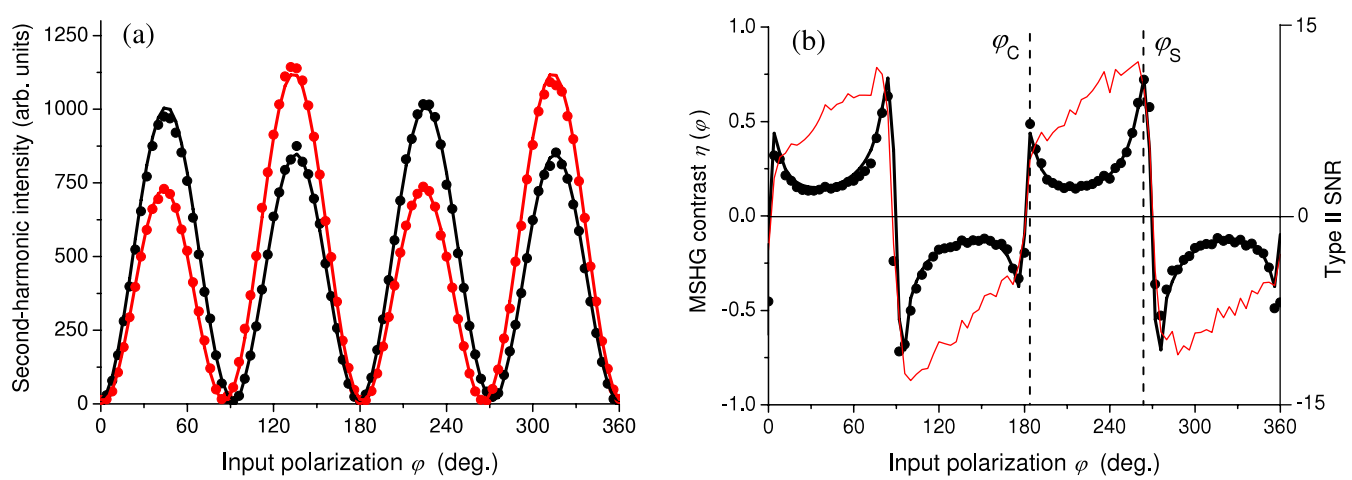

Figure 1. (a) NI y-polarized MSHG intensity at $80 \mathrm{~K}$ for $+M_{X}(\bullet)$ and $-M_{X}(\bullet)$ from a Au-capped 3 ML Fe film grown on vicinal W(110), using an applied magnetic field of $M_{X}= \pm 80 \mathrm{mT}$, with best fits to equation (2) (solid lines). (b) MSHG contrast (black dots) derived from (a), with a best fit (thick solid line) using equation (4), together with the Type II SNR (thin solid line), using equation (6). Examples of $\varphi_{\mathrm{c}}$ and $\varphi_{\mathrm{s}}$ are shown.

NI ensures no contribution to the even terms from the surface of the cap.

Based on both theory and experiment, a typical value of $\left|i j k L / i^{\prime} j^{\prime} k^{\prime}\right|$ is $\sim 0.1[11,20]$. Experimental data from a Au-capped $3 \mathrm{ML} \mathrm{Fe} \mathrm{film,} \mathrm{under} \mathrm{the} \mathrm{experimental} \mathrm{conditions}$ described in section 2, are shown in figure 1(a) (the small asymmetry between the opposite magnetizations arises from an imperfection in the output polarizer). Although the odd components, $y x x X M_{X}$ and $y y y X M_{X}$, are an order of magnitude less than the even component, $y x y$, they produce a large change in MSHG intensity on reversal of the interface magnetization. The values of $\varphi$ for which the largest relative changes in the MSHG intensity occur are determined from the MSHG contrast plot (also called an asymmetry plot):

$$
\eta_{y}(\varphi)=\frac{I_{y}\left(2 \omega ; \varphi,+M_{X}\right)-I_{y}\left(2 \omega ; \varphi,-M_{X}\right)}{I_{y}\left(2 \omega ; \varphi,+M_{X}\right)+I_{y}\left(2 \omega ; \varphi,-M_{X}\right)} .
$$

Substituting equation (2) into (3) gives

$$
\begin{aligned}
& \eta_{y}(\varphi) \\
& =\frac{2 \cos \delta|y x y \sin 2 \varphi|\left|\left(y x x X \cos ^{2} \varphi+y y y X \sin ^{2} \varphi\right) M_{X}\right|}{|y x y \sin 2 \varphi|^{2}+\left|\left(y x x X \cos ^{2} \varphi+y y y X \sin ^{2} \varphi\right) M_{X}\right|^{2}}
\end{aligned}
$$

where $\delta$ is the phase difference between the even crystallographic and odd magnetic contributions. Figure 1(b) illustrates the MSHG contrast plot of the figure 1(a) data. Equation (2) contains the symmetry $I_{y}(\pi+\varphi)=I_{y}(\varphi)$, and equation (3) $\eta_{y}(\pi-\varphi)=-\eta_{y}(\varphi)$. The contrast plots contain cusp-like turning points at the input polarization angles of $\varphi_{\mathrm{c}}$ and $\varphi_{\mathrm{s}}$, and symmetry-related positions. It has been shown that $\delta$ can be deduced from these cusps [17]. Also, measuring the $y$-polarized NI MSHG intensity near $\varphi_{\mathrm{c}}\left(\approx 0^{\circ}\right)$ or $\varphi_{\mathrm{s}}\left(\approx 90^{\circ}\right)$ reduces the odd contribution effectively to a single tensor component, simplifying the interpretation of results.

\section{Tuning the NI MSHG geometry to optimize the determination of hysteresis curves}

Two procedures for extracting magnetic hysteresis curves from the variation of MSHG intensity with applied magnetic field have recently been developed, which account properly for the quadratic term in the magnetization implicit in equation (2) [21]. This allows experimental geometries to be tuned to optimize the SNR of extracted hysteresis curves, without concern about loss of linearity as the applied magnetic field is varied [22]. The first of these procedures (Type I) can be used where the saturation magnetization for positive and negative magnetic fields is equal and opposite in magnitude, a very common property of hysteresis loops, including those of exchange-biased systems. Type I provides exact solutions around the cusp-like structures of figure 1(b) and, outside these regions, still provides a good approximation to the hysteresis loop [21]. The second technique (Type II) provides exact solutions for centrosymmetric hysteresis loops, which are also commonly observed, but this approach cannot be applied to exchange-biased interfaces. Centrosymmetric magnetization loops, where $M^{+}(H) \equiv-M^{-}(-H)$, are simply extracted using

$$
M^{ \pm}(H) \propto I^{ \pm}(2 \omega, H)-I^{\mp}(2 \omega,-H)
$$

where $M^{+}(H)$ has $H$ increasing from an initial negative value, and $M^{-}(H)$ has $H$ decreasing from an initial positive value, with $I^{+}(2 \omega, H)$ and $I^{-}(2 \omega, H)$ the corresponding MSHG intensities [21]. It will be shown later than Type II extraction can be extended to magnetically inhomogeneous interfaces.

The extracted hysteresis curve is expected to have a good SNR when the magnetic contrast is large, which occurs in the region of the cusp-like structures (figure 1(b)). The input polarization angle, $\varphi$, can be tuned to optimize the SNR in the extracted hysteresis curves. Provided the $\sqrt{N}$ noise of the $2 \omega$ photon counting is the dominant source of noise in the experiment, equation (5) leads to the following expression for the SNR:

$$
\begin{aligned}
& S_{y}(\varphi)=\frac{I_{y}\left(2 \omega ; \varphi,+M_{X}\right)-I_{y}\left(2 \omega ; \varphi,-M_{X}\right)}{\sqrt{I_{y}\left(2 \omega ; \varphi,+M_{X}\right)+I_{y}\left(2 \omega ; \varphi,-M_{X}\right)}} \\
& =\eta_{y}(\varphi) \sqrt{I_{y}\left(2 \omega ; \varphi,+M_{X}\right)+I_{y}\left(2 \omega ; \varphi,-M_{X}\right)} .
\end{aligned}
$$

The SNR behaviour is dominated by the shape of the magnetic contrast curve (figure 1(b)), and Type II extraction of these data achieves a SNR $>10$ for a significant ranges of $\varphi$ and is largest at $\varphi_{\mathrm{s}}$, where $\eta_{y}(\varphi)$ is a maximum. 

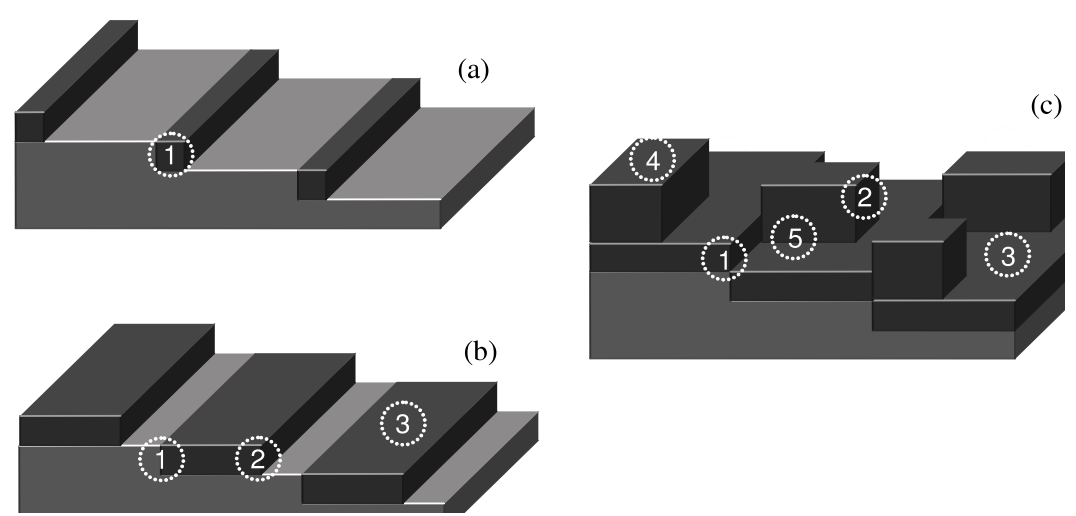

Figure 2. Schematic of some typical magnetic regions for nanostructures grown on vicinal interfaces (the capping layer is omitted for clarity). (a) Single atom wire interface $(n=1)$. (b) Sub-monolayer nanostripe interface $(n=3)$. (c) Stranski-Krastanov interface $(n=5)$.

Based on this analysis, the suggested methodology for optimizing the extraction of hysteresis curves is to measure the $y$-polarized NI magnetic contrast, $\eta(\varphi)$, as a function of input polarization angle, $\varphi$, to determine $\varphi_{\mathrm{c}}$ and $\varphi_{\mathrm{s}}$. The $y$ polarized NI MSHG intensity is measured close to $\varphi_{\mathrm{c}}$ or $\varphi_{\mathrm{s}}$ and the hysteresis curve can be extracted using both Type I and Type II techniques to determine whether the overall response is centrosymmetric [21]. If the two techniques produce the same extracted curves then Type II extraction can be extended to analyse the contributions from different magnetic regions to the overall response, as described below.

\section{Determining hysteresis loops from inhomogeneous low symmetry systems}

Buried magnetic interfaces, when formed by adsorption of magnetic material on non-magnetic substrates of $1 m$ symmetry and then capped by non-magnetic material, are generally inhomogeneous, with $n$ distinct magnetic regions, where the same magnetic species is found with different numbers of magnetic $\left(N_{\mathrm{M}}\right)$, substrate $\left(N_{\mathrm{S}}\right)$, and capping layer $\left(N_{\mathrm{C}}\right)$, nearest neighbours. For Fe deposition on $1.4^{\circ}$ vicinal W(110), it is well known that Fe nanostripes are formed at the step edges, with the width of the stripes increasing with further deposition until the (110) terraces are fully covered [8]. This growth mode continues to about 2 ML [23] but, at higher coverage, Stranski-Krastanov growth occurs, with large Fe islands being formed, at least ten atomic layers thick, on a pseudomorphic Fe monolayer [24].

Examples of some of the possible magnetic regions for this material system are shown in figure 2. In the limit, single atomic chains will be formed, where the number of distinct magnetic regions, $n=1$ (figure 2(a)). For submonolayer nanostripes, $n=3$ (figure 2(b)), and for StranskiKrastanov films, $n=5$ (figure 2(c)), assuming the large islands sit on a complete monolayer. Table 1 lists various regions, their local 2D symmetry, and values of $N_{\mathrm{M}}, N_{\mathrm{S}}$ and $N_{\mathrm{C}}$, assuming bcc structures with no reconstruction. The magnetic species in the different regions of the inhomogeneous interfaces in figure 2 have different values of these numbers. Both the magnetization [7, 25], and the nonlinear optical susceptibility [26], in the interfacial region are known to depend sensitively on nearest neighbour numbers, requiring
Table 1. Magnetic $\left(N_{\mathrm{M}}\right)$, substrate $\left(N_{\mathrm{S}}\right)$, and capping $\left(N_{\mathrm{C}}\right)$ atom nearest neighbour numbers for some capped bcc (110) nanostructures grown on a vicinal substrate of $1 \mathrm{~m}$ symmetry. The regions are identified in figure 2 .

\begin{tabular}{llllll}
\hline Interface type & Region & 2D symmetry & $N_{\mathrm{M}}$ & $N_{\mathrm{S}}$ & $N_{\mathrm{C}}$ \\
\hline Single atom chain & $(1)$ & $1 \mathrm{~m}$ & 2 & 3 & 3 \\
Sub-monolayer nanostripe & $(1)$ & $1 \mathrm{~m}$ & 3 & 3 & 2 \\
& $(2)$ & $1 \mathrm{~m}$ & 3 & 2 & 3 \\
\multirow{4}{*}{ Stranski-Krastanov } & $(3)$ & $2 \mathrm{~mm}$ & 4 & 2 & 2 \\
& $(1)$ & $1 \mathrm{~m}$ & 3 & 2 & 3 \\
& $(2)$ & $1 \mathrm{~m}$ & 6 & 0 & 2 \\
& $(3)$ & $2 \mathrm{~mm}$ & 4 & 2 & 2 \\
& $(4)$ & $2 m m$ & 6 & 0 & 2 \\
& $(5)$ & $2 m m$ & 6 & 2 & 0 \\
\hline
\end{tabular}

the contribution of the different regions to be included in the phenomenological model.

In developing a full model of $y$-polarized NI MSHG, symmetry considerations show that all $1 \mathrm{~m}$ and $2 \mathrm{~mm}$ regions can contribute to the odd magnetic contribution, but even crystallographic tensor components from regions of $2 \mathrm{~mm}$ symmetry are excluded, for substrates cut at small vicinal angles [16]. Assigning a local magnetization, $M_{X}^{(n)}(H)$, and a set of odd tensor components, $i j k L^{(n)}$, to each of the $n$ regions of the inhomogeneous interface, the intensity at $\varphi$ is given by

$$
\begin{aligned}
& I_{y}\left(2 \omega ; \varphi, M_{X}(H)\right) \propto \mid \sum_{n} y x y^{(n)} \sin 2 \varphi \\
& \quad+\left.\left(y x x X^{(n)} \cos ^{2} \varphi+y y y X^{(n)} \sin ^{2} \varphi\right) M_{X}^{(n)}(H)\right|^{2} .
\end{aligned}
$$

Fitting to this expression is impractical, given the number of independent tensor components, many of which may be complex. Very few MSHG studies of lower magnetic symmetry systems have been reported, due to this type of problem.

For centrosymmetric local magnetization loops, an extension of Type II curve extraction ([21] appendix B) gives:

$$
\begin{aligned}
& I^{ \pm}(2 \omega, H)-I^{\mp}(2 \omega,-H) \\
& \propto \sin 2 \varphi \cos ^{2} \varphi \sum_{n, n^{\prime}}\left|y x y^{(n)}\right|\left|y x x X^{\left(n^{\prime}\right)}\right| \\
& \quad \times \cos \left(\theta_{y x y}^{(n)}-\theta_{y x x X}^{\left(n^{\prime}\right)}\right) M_{X}^{\left(n^{\prime}\right) \pm}(H)
\end{aligned}
$$




$$
\begin{aligned}
& +\sin 2 \varphi \sin ^{2} \varphi \sum_{n, n^{\prime}}\left|y x y^{(n)}\right|\left|y y y^{\left(n^{\prime}\right)}\right| \\
& \times \cos \left(\theta_{y x y}^{(n)}-\theta_{y y y X}^{\left(n^{\prime}\right)}\right) M_{X}^{\left(n^{\prime}\right) \pm}(H)
\end{aligned}
$$

where $\theta_{i j k}^{(n)}$ is the complex phase factor, the summation includes $n=n^{\prime}$ terms, and the intensities have been defined in equation (5). If neither the absolute intensity nor phase is measured, $y x y^{(1)}$ can be set to unity and $\theta_{y x y}^{(1)}$ to zero. This subtraction procedure removes all terms quadratic in magnetization, including cross-terms between different magnetic regions, resulting in a much simplified equation that, for example, can be fitted to normalized sigmoidal magnetization loops of the form:

$$
M^{(n)+}(H)=-1+2 /\left\{1+\exp \left[-S^{(n)}\left(H-H_{\mathrm{c}}^{(n)}\right)\right]\right\}
$$

where $s^{(n)}$ is the softness and $H_{\mathrm{c}}^{(n)}$ the coercivity of the $n$th loop. Only the magnetization depends on the applied magnetic field in equation (8), with the remaining terms determining the scale of the measured response. In the absence of other information, this prevents the strength of the magnetization being determined, but the softness and coercivity are unaffected by these scaling factors. It can also be seen that measurements in the region of $\varphi_{\mathrm{c}}\left(\approx 0^{\circ}\right)$ and $\varphi_{\mathrm{s}} \quad\left(\approx 90^{\circ}\right)$ will produce a further simplification by restricting the odd term contribution to a single tensor component, $y x x X^{(n)}$ and $y y y X^{(n)}$, respectively, as mentioned above. Simulations confirm that such measurements maximize the probability of detecting the contribution from different magnetic regions. Finally, any significant magneto-elastic response (section 1) would be even in the magnetization, and the extracted curve (equation (8)) would then not be centrosymmetric.

MSHG thus offers the important diagnostic capability of exploiting the properties of the optical tensor components to identify different magnetization contributions from inhomogeneous interfaces, because the components will vary with the local atomic structure (figure 2 and table 1). The dispersive behaviour of these components will also vary, allowing the response of different regions to be optimized by scanning the excitation wavelength. Standard methods of measuring thin film magnetization do not offer this important capability of varying the contribution from different magnetic regions to the total signal. Of course, it remains possible that the contribution from some regions may be too small to be detected. It is also possible that the regions could have different easy axes of magnetization, but this can be tested by changing the experimental geometry, and appropriate expressions can be derived for perpendicular magnetization, for example [16].

\section{Hysteresis loops from Au-capped Fe nanostructures grown on vicinal W(110)}

As discussed above, for Fe deposition on $1.4^{\circ}$ vicinal W(110), Fe nanostripes are formed at the step edges, with the width of the stripes increasing with further deposition until the (110) terraces are covered by continuous, pseudomorphic Fe monolayers [8]. This growth mode continues to about
Table 2. Fitted values of the coercivity $\left(H_{\mathrm{c}}\right)$ and softness $(s)$ of the hysteresis loops (equations (9)). The estimated error in the last figure is given in parenthesis.

\begin{tabular}{llllll}
\hline & \multicolumn{2}{c}{ 1st region } & & \multicolumn{2}{c}{ 2nd region } \\
\cline { 2 - 3 } \cline { 6 - 6 } Sample & Coercivity (mT) & Softness & & Coercivity (mT) & Softness \\
\hline $0.25 \mathrm{ML} \mathrm{Fe}$ & $2.9(5)$ & $0.07(1)$ & - & - \\
$0.75 \mathrm{ML} \mathrm{Fe}$ & $49(3)$ & $0.08(1)$ & & $13(2)$ & $0.12(1)$ \\
$2 \mathrm{ML} \mathrm{Fe}$ & $28.8(1)$ & $0.43(1)$ & - & - \\
$3 \mathrm{ML} \mathrm{Fe}$ & $37.8(3)$ & $0.17(1)$ & $18.5(2)$ & $0.25(1)$ \\
\hline
\end{tabular}

2 ML [23] but, by 3 ML, Stranski-Krastanov growth is established, with large $\mathrm{Fe}$ islands being formed, at least ten atomic layers thick, on a pseudomorphic Fe monolayer [24]. It is well established that capping such Fe layers with $\mathrm{Au}$ at room temperature does not produce any inter-diffusion or reaction [9]. The left panels of figure 3 show pairs of MSHG intensity measurements at $80 \mathrm{~K}$ from $0.25,0.75,2$ and $3 \mathrm{ML}$ Fe structures, obtained at angles close to $\varphi_{\mathrm{c}}\left(\approx 0^{\circ}\right)$ and $\varphi_{\mathrm{s}}\left(\approx 90^{\circ}\right)$. The right panels of figure 3 show the curves resulting from using Type II extraction (equation (8)), together with simultaneous nonlinear least squares fits (solid lines) to the two data sets using one or two sigmoidal loops (equations (9)), as required by the data.

A number of conclusions can be drawn immediately from figure 3. Firstly, the new, optimized procedure is sensitive to at least $0.25 \mathrm{ML} \mathrm{Fe}$ (figure 3(a)), with unamplified laser pulses. Secondly, the MSHG data are not easy to interpret prior to Type II extraction: curves can point in either direction, depending on the value of the complex phase, $\delta$, and are offset from zero by an amount determined by the relative size of the crystallographic (even) and magnetic (odd) terms [21]. Thirdly, the right panel $2 \mathrm{ML} \mathrm{Fe}$ result (figure 3(c)) shows that the extracted $\varphi_{\mathrm{c}}$ and $\varphi_{\mathrm{s}}$ curves overlap exactly when scaled to the same saturation value, if only one region is contributing to the response (consistent with equation (8)). Fourthly, the extracted curves are centrosymmetric within experimental error, thus excluding any significant magnetoelastic contribution.

The SNR allows two regions to be identified for the $0.75 \mathrm{ML} \mathrm{Fe}$ and $3 \mathrm{ML} \mathrm{Fe}$ data. The magnetization axis scales with the experimental data, but is otherwise arbitrary, as discussed in the previous section. In two cases the scaling has been changed, as indicated in the figure, to aid the discussion. The hysteresis loop parameters determined from the fits to the curves are listed in table 2 and the normalized loops are shown in figure 4. Figure 4(d) also shows the average total loop that would be observed for 3 ML Fe by magnetometry assuming, for each loop, the same saturation magnetization.

Comparison of the left and right panels shows that smaller variations in the MSHG intensity with applied magnetic field result in a poorer SNR in the extracted curves. However, this optimized approach to the measurement and extraction of magnetic information can obtain useful information even from 0.25 ML Fe stripes which, for this $1.4^{\circ}$ offcut, are an average of 10 atoms wide, with a $30 \%$ width distribution [8]. Figures 3(a) and 4(a) show ferromagnetic behaviour, but 

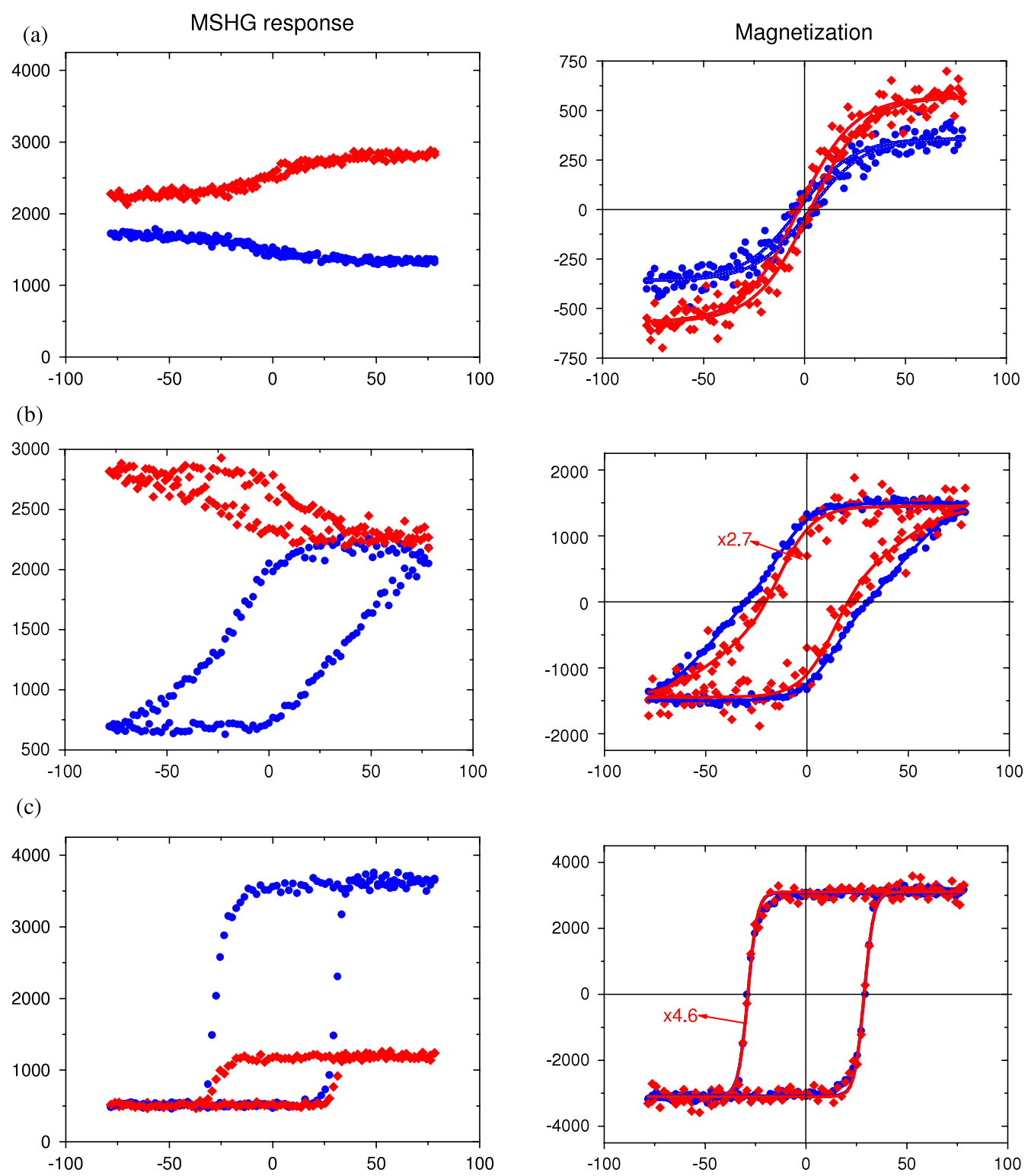

(d)
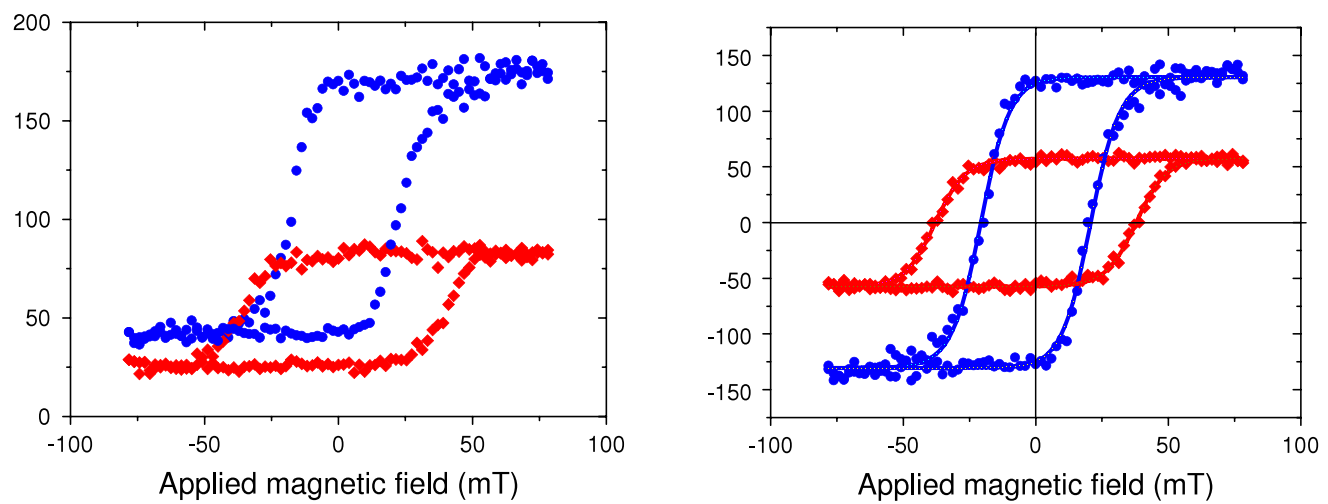

Figure 3. NI MSHG $y$-polarized intensity (left panel) at $80 \mathrm{~K}$ and (right panel) Type II extracted hysteresis curves with sigmoidal loop fits (solid lines), from Au-capped Fe films grown on vicinal W(110): $\varphi \approx \varphi_{\mathrm{s}}(\bullet), \varphi \approx \varphi_{\mathrm{c}}$ (४); (a) $0.25 \mathrm{ML}$, (b) $0.75 \mathrm{ML}$, (c) $2 \mathrm{ML}$, (d) $3 \mathrm{ML}$ Fe.

only one loop is required to fit the data, even though three distinct regions could contribute to the magnetization for these capped sub-monolayer nanostripes (figure 2(b) and table 1). Previous work on this vicinal system has indicated that, for the uncapped structure, ferromagnetism occurs in stripes of 4 atoms width and greater [6]. Although ferromagnetic behaviour is observed here, both lower temperature studies and perpendicular magnetization measurements, which are not 
(a)

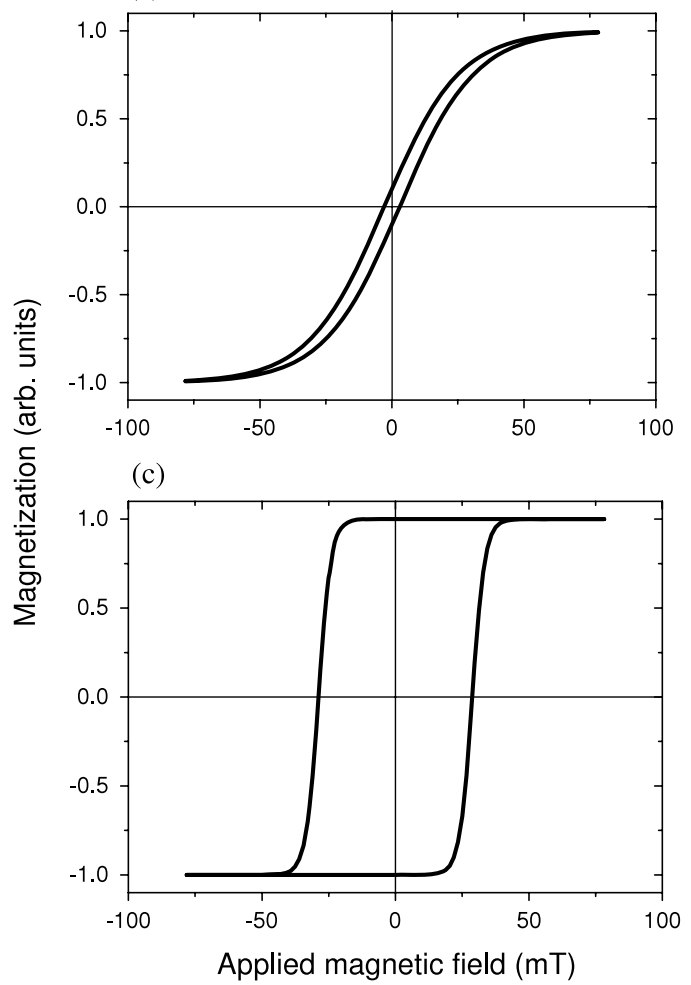

(b)

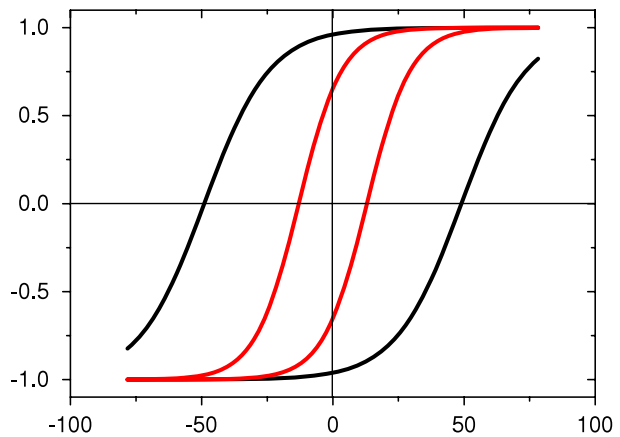

(d)

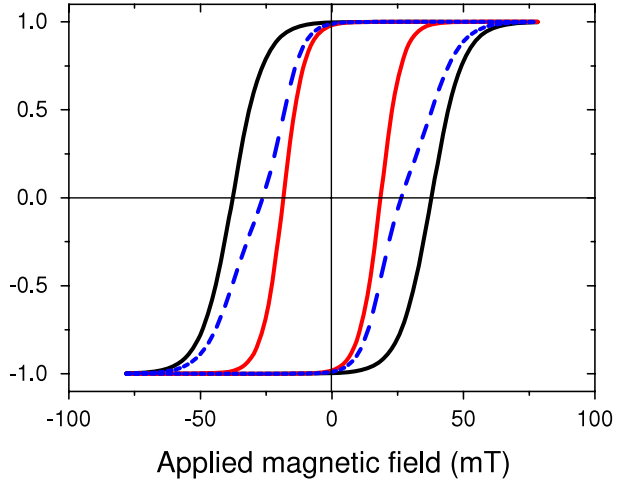

Figure 4. Normalized hysteresis loops from the fits of figure 3 (solid lines): (a) 0.25 ML (b) 0.75 ML (c) 2 ML (d) 3 ML Fe, where the dashed line is the average total response, assuming the same saturation magnetization for each loop.

possible with the current experimental arrangement, would be needed for more detailed interpretation. For example, it is possible that the steps (figure 2(b), regions 1 and 2) have an out-of-plane easy axis [2], or a lower Curie temperature $\left(T_{\mathrm{C}}\right)$ due to increased instability to magnon formation as dimensionality is reduced [27].

Figures 3(b) and 4(b) show at least two regions contributing to the magnetization of the capped $0.75 \mathrm{ML}$ Fe nanostripes (the $\varphi \approx \varphi_{\mathrm{c}}$ curve is scaled by $2.7 \times$ for clarity). Previous results from this vicinal system, using the magneto-optic Kerr effect (MOKE), for 0.7 ML Fe capped by $6 \mathrm{ML} \mathrm{Au}$, show a single loop of coercivity $30 \mathrm{mT}$ [9]. MOKE will measure the average response from the step and terrace atoms [28] and, for this offcut and coverage, step atoms will contribute only $\sim 4 \%$ of the signal: the MOKE loop is dominated by the terrace magnetization for this low angle offcut. In contrast, SHG can be disproportionately sensitive to step structure [10], and it is suggested that this leads to the detection of both terrace (figures 2(b), region 3 ) and step magnetization (regions 1 and 2) in the $0.75 \mathrm{ML}$ sample. The terrace coercivity of $49 \mathrm{mT}$ is larger than the $30 \mathrm{mT}$ measured previously [9]. However, the capping layers are of very different thicknesses (6 and $60 \mathrm{ML}$ ) and this will affect the oscillatory interlayer Ruderman-KittelKasuya-Yosida (RKKY) exchange coupling [29] and thus the coercivity and $T_{\mathrm{C}}$ [30]. A different coercivity, arising from a different capping layer thickness, is thus possible.

The second region detected is likely to be that of the $\mathrm{Au}$ capped Fe step (figure 2(b), region 2). Previous work has shown that the effect of capping nanostripes of this width with $\mathrm{Au}$ is to raise the apparent coercivity from $5 \mathrm{mT}$ [8] to $30 \mathrm{mT}$ [9] and the measured $T_{\mathrm{C}}$ from $\sim 185 \mathrm{~K}$ [8] to $\sim 225 \mathrm{~K}$ [31]. It thus appears likely that the $\mathrm{Fe}$ atoms at the $\mathrm{W}(110)$ step (figure 2(b), region 1 ), which have $1 / 3$ rd fewer Au nearest neighbours than the Au-capped Fe step, will make the smaller contribution of the two step regions to the magnetic response.

Figure 3(c) shows a single region contributing to the magnetization of the capped 2 ML Fe layer, as discussed above. The hysteresis loop (figure 4(c)) is reasonably hard (table 2), and the saturation and remanence are the same, confirming the [110] easy axis. The W/Fe and Fe/Au terrace interfaces of the $2 \mathrm{ML}$ thick Fe film are not distinguished, which is understandable given the extended character of electronic wavefunctions and calculations that show significant magnetic interactions extending 3 atomic layers into the substrate for $\mathrm{Fe}$ bilayers on $\mathrm{W}(110)$ [32]. The terraces dominate the response of the $\mathrm{W} / \mathrm{Fe} / \mathrm{Au}$ interfacial region, as no signature of the $\mathrm{Fe}$ steps could be extracted, in contrast to the $0.75 \mathrm{ML} \mathrm{Fe}$ results.

As discussed above, Stranski-Krastanov growth is expected for $3 \mathrm{ML}$ Fe coverage, with large Fe islands being formed, at least ten atomic layers thick, on a pseudomorphic Fe monolayer [24]. Figure 3(d) shows that two distinct curves are observed, with both loops showing [110] easy axes (figure 4(d)), in agreement with previous conclusions that the easy axes of the islands and the monolayer are aligned [24]. As figure 2 shows, there are 5 distinct regions that may contribute to the MSHG response (it is assumed that the island interface 
with the pseudomorphic Fe monolayer cannot be distinguished from the $\mathrm{Fe} / \mathrm{W}$ interface response, one atomic layer below). Of the 5 regions, the step response from regions 1 and 5 will be small, based on the $2 \mathrm{ML}$ result for the $\mathrm{W} / \mathrm{Fe} / \mathrm{Au}$ interfacial region. For region 2, strong cancellation in the signal from opposite edges of the islands will occur, due to phase reversal. The magnetic dipolar response from the bulk of the large Fe islands is excluded by symmetry. The two loops observed must come from the remaining regions 3 and 4, the $\mathrm{W} / \mathrm{Fe} / \mathrm{Au}$ and $\mathrm{Fe} / \mathrm{Au}$ terrace interfaces. As the Fe monolayer is still pseudomorphic and strained, it is likely that the larger coercivity of $39 \mathrm{mT}$ is associated with the W/Fe/Au terrace interface, although this is lower than the $0.75 \mathrm{ML}$ value of $49 \mathrm{mT}$. The Fe/Au interface is likely to be relatively unstrained, as the tensile strain will be reduced on island formation and $\mathrm{Au}$ growth on $\mathrm{Fe}(110)$ is largely unstrained $[18,33]$. The lower coercivity of $18 \mathrm{mT}$ may be a consequence of the reduced strain. Figure 4(d) shows that the average response, assuming the same saturation magnetization, has a coercivity of $26 \mathrm{mT}$, similar to the $2 \mathrm{ML} \mathrm{Fe}$ value of $29 \mathrm{mT}$, where the interfaces are not distinguished. The two interfaces may have a similar saturation magnetization: a $29 \mathrm{mT}$ coercivity is obtained by combining the two loops, if the saturation magnetization of the narrower loop is reduced by $25 \%$.

\section{Conclusion}

The variation in MSHG tensor components with the local atomic structure can, in principle, be exploited to identify different magnetization contributions from inhomogeneous interfaces. However, the additional information available, compared to more commonly used techniques like MOKE, produces a more complex response that can be difficult to interpret, particularly for low symmetry structures. An experimental procedure and phenomenology has been developed that uses normal incidence geometry to simplify the MSHG response from aligned magnetic nanostructures grown on low symmetry substrates. Where the hysteresis loops are centrosymmetric, the curve extraction technique eliminates quadratic and cross-terms in the magnetization, thus simplifying analysis and also allowing the SNR to be optimized, which occurs near input polarization angles of $0^{\circ}$ and $90^{\circ}$ (and symmetry-related positions). Measuring close to these two angles also increases the probability of detecting the contribution from the distinct regions of an inhomogeneous magnetic system by maximizing the difference in the magnetic tensor component contributions. This new approach has been applied to a well-known system, Fe nanostripes grown on vicinal W(110), capped with Au. The increased sensitivity allows a hysteresis loop from 10 atom wide Fe nanostripes to be detected, using unamplified fs laser pulses. Two different hysteresis loops have been identified for the W/Fe/Au and $\mathrm{Fe} / \mathrm{Au}$ interfacial regions of the $\mathrm{Fe}$ islands, produced by Stranski-Krastanov growth at $3 \mathrm{ML}$ Fe coverage, while only a single interfacial region and loop is detected for the layerby-layer growth at $2 \mathrm{ML}$ Fe. The results for $0.75 \mathrm{ML}$ $\mathrm{Fe}$ coverage are of particular interest, as contributions from terrace and step Fe atoms have been distinguished. This experimental procedure and phenomenology opens up low symmetry magnetic interfaces and aligned nanostructures to characterization by MSHG.

\section{Acknowledgments}

This publication has emanated from research conducted with the financial support of Science Foundation Ireland, the Irish Higher Education Authority and the Irish Research Council for Science, Engineering and Technology.

\section{References}

[1] Gambardella P, Dallmeyer A, Malti K, Malagoli M C, Eberhardt W, Kern K and Carbone C 2002 Nature 416301

[2] Gambardella P, Dallmeyer A, Maiti K, Malagoli M C, Rusponi S, Ohresser P, Eberhardt W, Carbone C and Kern K 2004 Phys. Rev. Lett. 93077203

[3] McGilp J F 1990 J. Phys.: Condens. Matter 27985

[4] Kirilyuk A and Rasing T 2005 J. Opt. Soc. Am. B 22148

[5] Sampaio L C, Mougin A, Ferre J, Georges P, Brun A, Bernas H, Poppe S, Mewes T, Fassbender J and Hillebrands B 2003 Europhys. Lett. 63819

[6] Elmers H J, Hauschild J, Hoche H, Gradmann U, Bethge H, Heuer D and Kohler U 1994 Phys. Rev. Lett. 73898

[7] Elmers H J 1995 Int. J. Mod. Phys. 93115

[8] Hauschild J, Elmers H J and Gradmann U 1998 Phys. Rev. B 57 R677

[9] Pratzer M and Elmers H J 2003 Phys. Rev. B 6794416

[10] Power J R, O’Mahony J D, Chandola S and McGilp J F 1995 Phys. Rev. Lett. 751138

[11] Pan R-P, Wei H D and Shen Y R 1989 Phys. Rev. B 391229

[12] Jeong J-W, Shin S-C, Lyubchanskii I L and Varyukhin V N 2000 Phys. Rev. B 6213455

[13] Callen E R and Callen H B 1963 Phys. Rev. 129578

[14] du Tremolet de Lacheisserie E 1995 Phys. Rev. B 5115925

[15] Shen Y R 1984 The Principles of Nonlinear Optics (New York: Wiley)

[16] Carroll L and McGilp J F 2003 Phys. Status Solidi c 03046

[17] Carroll L, Fleischer K and McGilp J F 2008 Phys. Status Solidi $\mathrm{c}$ at press

[18] Fleischer K, Carroll L, Smith C and McGilp J F 2007 J. Phys.: Condens. Matter 19266003

[19] Dewitz J P, Jian C and Hubner W 1998 Phys. Rev. B 585093

[20] Hubner W and Bennemann K H 1989 Phys. Rev. B 405973

[21] McGilp J F, Carroll L and Fleischer K 2007 J. Phys.: Condens. Matter 19396002

[22] Valev V K, Gruyters M, Kirilyuk A and Rasing T 2005 Phys. Status Solidi b $\mathbf{2 4 2} 3027$

[23] Hauschild J, Gradmann U and Elmers H J 1998 Appl. Phys. Lett. 723211

[24] Bode M, Wachowiak A, Wiebe J, Kubetzka A, Morgenstern M and Wiesendanger R 2004 Appl. Phys. Lett. 84948

[25] Repetto D, Lee T Y, Rusponi S, Honolka J, Kuhnke K, Sessi V, Starke U, Brune H, Gambardella P, Carbone C, Enders A and Kern K 2006 Phys. Rev. B 7454408

[26] Jin Q Y, Regensburger H, Vollmer R and Kirschner J 1998 Phys. Rev. Lett. 804056

[27] Bobo J F, Gabillet L and Bibes M 2004 J. Phys.: Condens. Matter 16471

[28] Bader S D 2002 Surf. Sci. 500172

[29] Grunberg P, Schreiber R, Pang Y, Brodsky M B and Sowers H 1986 Phys. Rev. Lett. 572442

[30] Vollmer R, van Dijken S, Schleberger M and Kirschner J 2000 Phys. Rev. B 611303

[31] Carroll L, Fleischer K and McGilp J F 2008 at press

[32] Qian X and Hubner W 1999 Phys. Rev. B 6016192

[33] Elmers H J and Gradmann U 1994 Surf. Sci. 304201 